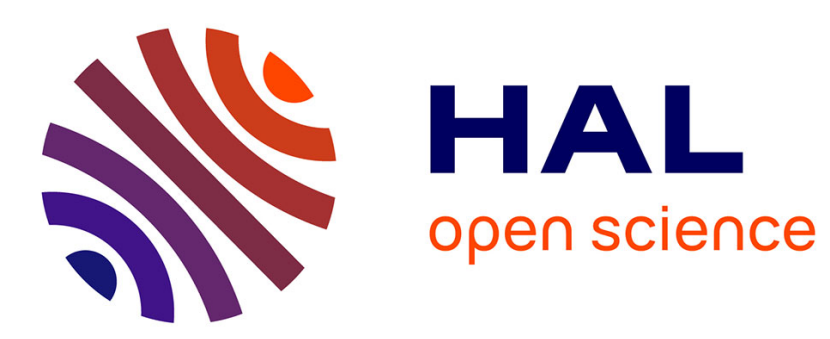

\title{
Inductive reasoning, food neophobia, and domain-specificity in preschoolers
}

Camille Rioux, Lucas Leglaye, Jérémie Lafraire

\section{To cite this version:}

Camille Rioux, Lucas Leglaye, Jérémie Lafraire. Inductive reasoning, food neophobia, and domain-specificity in preschoolers. Cognitive Development, 2018, 47, pp.124 - 132. 10.1016/j.cogdev.2018.05.001 . hal-03306895

\section{HAL Id: hal-03306895 \\ https://hal.science/hal-03306895}

Submitted on 29 Jul 2021

HAL is a multi-disciplinary open access archive for the deposit and dissemination of scientific research documents, whether they are published or not. The documents may come from teaching and research institutions in France or abroad, or from public or private research centers.
L'archive ouverte pluridisciplinaire HAL, est destinée au dépôt et à la diffusion de documents scientifiques de niveau recherche, publiés ou non, émanant des établissements d'enseignement et de recherche français ou étrangers, des laboratoires publics ou privés. 
Inductive reasoning, food neophobia, and domain-specificity in preschoolers

\author{
Camille Rioux $^{a, b^{*}}$, Lucas Leglaye ${ }^{a}$ \& Jérémie Lafraire ${ }^{a}$
}

${ }^{a}$ Center for Food and Hospitality Research, Paul Bocuse Institute, Ecully, France

${ }^{\mathrm{b}}$ Aix Marseille Université, PSYCLE EA3273, 13621 Aix en Provence, France

(C) 2018. This manuscript version is made available under the CC-BY-NC-ND 4.0 license http://creativecommons.org/licenses/by-nc-nd/4.0/

\title{
This manuscript is published in Cognitive Development
}

Rioux, C., Leglaye, L., \& Lafraire, J. (2018). Inductive reasoning, food neophobia, and domain-specificity in preschoolers. Cognitive Development, 47, 124-132. https://doi.org/10.1016/j.cogdev.2018.05.001 


\begin{abstract}
The present study aimed at unravelling the previously reported negative relationship between inductive reasoning performance and the intensity of food rejection tendency (namely food neophobia and pickiness) in preschoolers. More precisely, the objective of the present study is to determine whether the previously reported correlation between poor inductive reasoning performance and high food rejection score is restricted to the food domain only or extends to other domains (e.g. the artefact domain).
\end{abstract}

To address these questions, we tested 109 preschoolers (mean age $=46.90$ months). A food rejection score was computed beforehand with a hetero-assessment scale for each subject. Then, the children were asked to perform an induction task followed by a discrimination task on food and artefact stimuli. Results revealed that neophobic children were characterized by poorer induction performance in both the food and the artefact domain compared to their neophilic counterparts. In addition, our results support the hypothesis that inductive reasoning on food stimuli exhibits some sign of domain-specificity. We conclude that the results of the present study pave the way to evidence-based interventions in public health tailored to the specificities of preschoolers' early food categories.

Keywords: preschoolers, food rejection, inductive reasoning, domain-specificity 


\section{Introduction}

The view that human beings are only endowed with domain general reasoning abilities they could use on any cognitive task whatever its structure and its content has been seriously challenged over the past decades. Indeed, originated in the pioneer work of Chomsky (1980), Marr (1982) and Fodor (1983), the idea that we are equipped with specialized and constrained learning mechanisms dedicated to particular domains of knowledge has received a great deal of attention for a variety of both theoretical and empirical reasons (Hirschfield and Gelman, 1994, pp. 3-36 for an overview). Among these reasons, hypothesizing domainspecific constrains on learning mechanisms appeared as the key to solve the problem of induction (i.e., the same experience is potentially relevant to extremely diverse inductions, Gelman, 1990). Indeed one way to make the problem of induction easier is to put domain specific constraints on the learning mechanisms to narrow the range of inputs a specific mechanism is sensitive to, and narrow the range of possible interpretations of these inputs (Keil, 1981). Since the problem of induction has to be addressed when it comes to investigate conceptual development in children, many domains of knowledge have been deeply and fruitfully scrutinized over the past decades (e.g., the domain of physics or number, Spelke \& Kinzler, 2007). However, despite the wealth of research on domain specificity, certain very plausible candidates to the status of specific domains of knowledge have been underresearched so far.

\section{Domain specificity and the food domain}

The food domain partly felt out of the scope of the domain approach. Perhaps one of the reasons why it has been partly neglected is because food items are borderline cases with respect to the distinction between natural kinds and artifacts (Rumiati \& Foroni, 2016) which has been, on the contrary, extensively studied (Gelman, 1988, 2004). In the following 
sections, we will briefly review both theoretical and empirical reasons to fill this research gap.

First there is a theoretical reason to consider the food domain as a specific domain of knowledge. Indeed, a domain of knowledge is generally characterized as an adaptation to a range of phenomena that presented problems in the ancestral environment (Sperber \& Hirschfeld, 2004) and it is argued that the information in the brain is represented in domains that are important for the survival and fitness of individuals (Mahon \& Caramazza, 2011). Interestingly, there was such a problem related to human foraging behavior: the omnivore dilemma. As insightfully stated by Rozin (1976), "trying new foods is at the core of omnivorousness (. . ) so is being wary of them". In a nutshell, after weaning children need to enlarge their dietary repertoire to satisfy their nutritional needs. They must therefore continually sample new food resources as they move away from maternal milk. However, this search for variety can prove hazardous, as new substances may be toxic, and a single mistake in this search could potentially lead to death, and thus hinder fitness (associated with evolutionary success; Dawkins, 1976). Therefore determining which foods are edible and which ones are toxic in a local environment has been an essential task throughout human evolution and may have shaped the way we process information in the food domain (Wertz \& Wynn, 2014a; 2014b).

Corroborating this hypothesis, one important empirical finding in the food domain is that children from the age of 2 to 3 years attend to information about color or texture, rather than shape, when discriminating between edible and inedible substances or between different kinds of foods, and also when it comes to generalize novel word and properties to food versus artifacts (Lavin \& Hall, 2001; Macario, 1991). Moreover, a recent study has shown that 
humans tend to rely on color to categorize natural foods according to their energetic value (Foroni, Pergola, \& Rumiati, 2016, Foroni \& Rumiati, 2017). More precisely, subjects were biased toward attributing significantly less energy to greener food than red food, even when actual calorie content was controlled for. More reddish nuances in natural food items (e.g., fruits) do generally indicate higher energy or greater protein contents (Foroni et al., Ibidem), therefore being able to identify food items by color would constitute an evolutionary advantage. Furthermore, a recent study conducted by Rioux, Picard, \& Lafraire (2016) shed new light on the nature of the contribution of color cues to food categorization in preschoolers. In a discrimination task involving super-ordinate categories (vegetable vs. fruit) color typicality impacted the children's discrimination performances (performances were better for typically colored than for atypically colored food items). Thus, the contribution of color cues in the food domain seems at least twofold: certain colors do serve as proxies of energy density but also convey information regarding the typicality of certain food items which in turn may foster or hinder food categorization at least in preschoolers.

These pieces of evidence suggest that color has a special predictive validity in the food domain. The range of inputs food categorization is sensitive to, is constrained in that food categorization may exhibit some specific color-dependence not observable in other domains. One of the ambitions of the present study is to determine whether this is also the case of inductive reasoning about food $(\mathrm{H} 1)$.

\section{The relationship between food categories and food rejection}

One feature of a domain of knowledge is that it is generally a stable answer to a recurring and complex problem in the ancestral environment (Sperber \& Hirschfeld, 2004). The main problem connected to foraging is the omnivore dilemma. We have seen in the previous section that a categorization system constrained to be more sensitive to color information is 
one response to this adaptive problem. It underpins crucial functions such as discriminating between edible versus non-edible substances, and generalizing for instance the edibility property to exemplars of the same food category (see Murphy, 2002, p.3 for an interesting thought experiment). However, even if we acknowledge that the specific food categorization system and the capacities it underpins constitute a response to the omnivore problem, it grasps only one horn of the dilemma. Indeed, a protective system against the ingestion of potentially dangerous substances (berries and plants in the hunter-gatherer environment) is also needed. Food rejection, particularly food neophobia, has been presented as such a protective and therefore adaptive mechanism (Gigerenzer \& Goldstein, 2011; Rozin, 1979). The notion of food rejection encompasses two distinct, though correlated, dimensions: food neophobia and food pickiness (Lafraire, Rioux, Giboreau, \& Picard, 2016; Rioux, Lafraire, \& Picard, 2017a). Food neophobia is generally defined as the reluctance to eat novel food (Birch \& Fischer, 1998; Pliner \& Hobden, 1992) whereas food pickiness concerns both familiar and unfamiliar food items (Taylor, Wernimont, Northstone, \& Emmett, 2015). Food rejection has been presented as one of the strongest psychological barriers to a diversified diet, as it mostly targets fruits and vegetables (Dovey, Staples, Gibson, \& Halford, 2008; Falciglia, Couch, Gribble, Pabst, \& Frank, 2000).

An interaction between food rejection and the food categorization system has been empirically revealed recently. A first experiment conducted with children from 3 to 5 years of age, revealed that the intensity of food rejection was negatively correlated with children's performances on a food categorization task (Rioux et al., 2016). Such a negative correlation has been observed also between the intensity of food rejection and preschooler's performance on an induction task on food stimuli (Rioux, Lafraire, \& Picard, 2017b). Regarding the direction of the causal arrow, the authors suggested that we could be facing a vicious circle. 
Food rejection may hinder the development of the food categories. Reciprocally, a child with poor conceptual knowledge about food encounters difficulties recognizing various instances of the same categories and consequently is more prone to reject them. Similarly, a child with poor inductive reasoning abilities encounters difficulties generalizing properties (e.g. the edibility property) to food items and consequently is more prone to reject them.

However, these studies suffered from some limitations. In particular, the children were always asked to perform categorization and induction tasks on food stimuli only. Whether the reported relationship between food rejection and categorization and induction performance is specific to the food domain or is a domain general association (i.e. for whatever domain early categorization and induction abilities are applied to), is a question that has been left unresolved. Actually, if the response to the specific problem an omnivorous has to deal with in foraging tasks is what counts as the specific domain of knowledge, and if the response is twofold since it embeds the food categorization system and food rejection, then the relationship itself between food rejection and the food categories system might be the locus of domain specificity. This is precisely the second hypothesis we tested in the present experiment $(\mathrm{H} 2)$.

\section{The Present Study}

We thus designed the present study to test the following hypotheses:

H1 Early inductive reasoning strategies are based on color in the food domain and based on shape in the artifact domain.

$\mathrm{H} 2$ Food rejection is correlated to both discrimination and induction abilities in the food domain but not in the artifact domain. 
We first collected individual scores on the Children Food Rejection Scale (CFRS, Rioux et al., 2017a). Next, to measure the children's inductive reasoning abilities in the food and artifact domains, the participants were asked to complete a property generalization task (induction task), in which perceptual and categorical pieces of information were pitted against each other (Gelman \& Markman, 1986; see Rioux et al., 2017b for a similar design in the food domain). The participants completed the property generalization task on both food and artifact stimuli. Then the participants were asked to complete a forced sorting task (discrimination task) whose design was similar to that on food stimuli by Rioux et al. (2016).

\section{Method}

\section{Participants}

Preschoolers participated to the study $(n=109,52$ girls and 57 boys; age range $=39-56$ months; mean age $=46.90$ months; $s d=4.23$ ). This age range was selected since food rejection behaviors occur at this age period (Dovey et al., 2008; Lafraire et al., 2016). Children were predominately Caucasian and came from middle-class communities. They were all pupils in preschools located in Lyon and Paris conurbations. Prior the study, caregivers filled out the Children Food Rejection Scale (CFRS, Rioux, et al, 2017a). The CFRS includes two subscales corresponding to two dimensions of food rejection, food neophobia (6 items) and pickiness (5 items). Caregivers were asked to what extent they agree with statements regarding their child's neophobia (e.g. "My child is constantly looking for familiar foods") and pickiness ("My child refuses certain foods due to their texture"). Caregivers rated each item on a 5-point Likert-like scale (Strongly disagree, Disagree, Neither agree nor disagree, Agree, Strongly agree). Each answer was then numerically coded with high scores indicating higher food neophobia or pickiness (scores 
could range from 6 to 30 for neophobia, 5 to 25 for pickiness; food neophobia scores: $M=$ $16.86, s d=5.58$; food pickiness scores: $M=17.94, s d=4.09$ ). Within this young population, only a hetero-assessment scale can be used (caregivers respond on behalf of their children). Indeed, toddlers have difficulty responding on a several-point agreement scale (Guignard, 2000; Laureati, Bergamaschi, \& Pagliarini, 2015). While it is important to have in mind that hetero-assessment scales raise several difficulties (Laureati et al., 2015) and provide only an approximation of children behaviors (Pliner, 1994), caregivers reports of their children's food neophobia and pickiness using the CFRS, have be shown to correlate with actual neophobic and picky behaviors of children (Rioux et al., 2017a).

\section{Materials and procedure}

\section{Induction Task}

The materials consisted of 14 triad sets with photographs of unknown fruits, vegetables, artifacts (pieces of tools without any salient functional properties) and minerals, and were controlled for size, contrast, and luminosity. Each triad, printed on a laminated card measuring 21 x $29.7 \mathrm{~cm}$, contained three pictures: one target picture and two test pictures. Test picture 1 belonged to the same taxonomic superordinate category as the target picture, but was perceptually dissimilar, and test picture 2 belonged to a different taxonomic superordinate category from the target picture, but was perceptually similar. For instance, in Figure 1a, the target picture is a fruit (a java apple), test picture 1 is another fruit dissimilar in color and shape (a pitaya), and test picture 2 is a mineral similar in color (a crocoite crystal). The three pictures used in each set in the present study were different across the 14 triads. 
Figure 1. Example of triads used in the induction task.

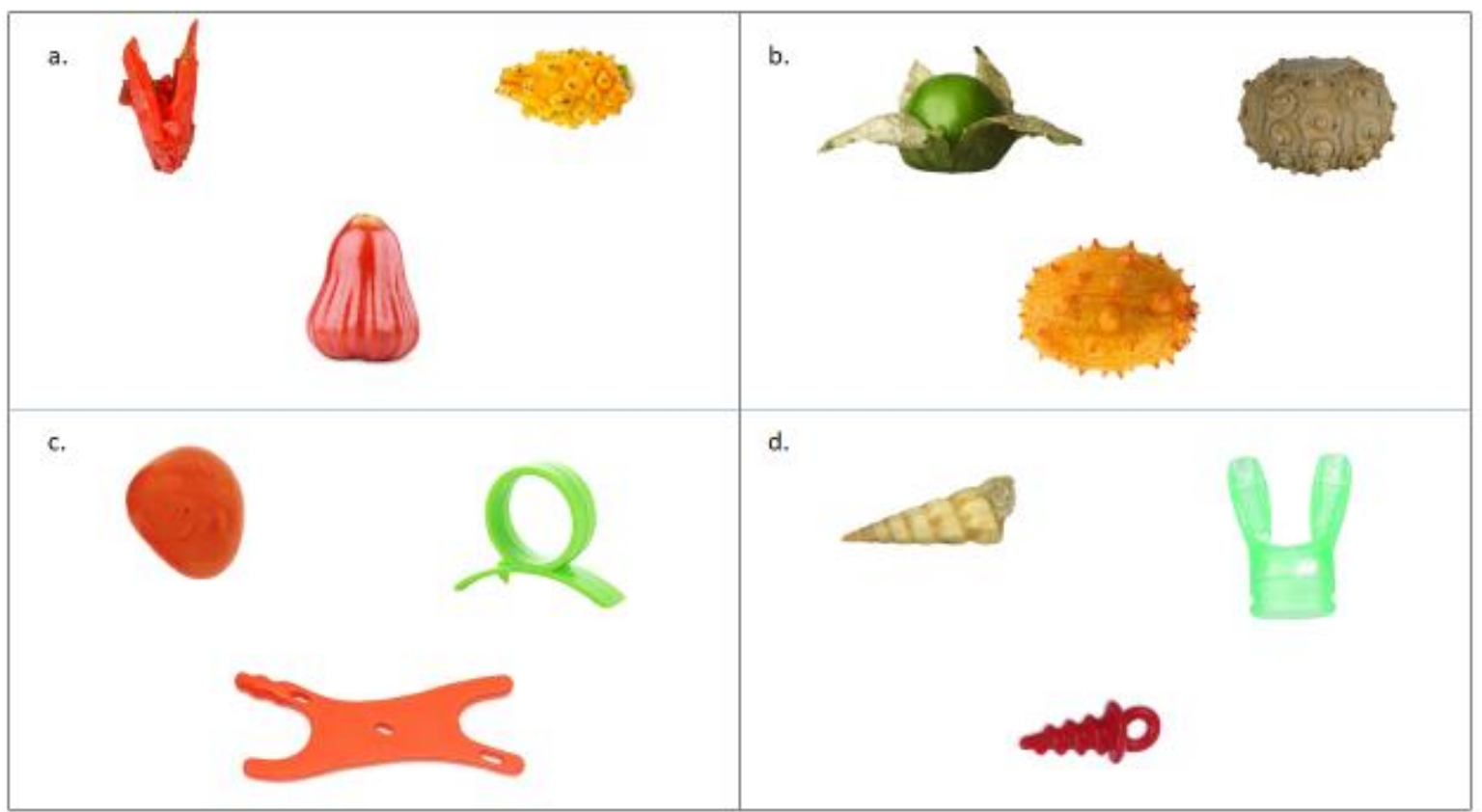

Note. (a) Food-color triad, (b) Food-shape triad, (c) Artifact-color triad, (d) Artifact-shape triad. Target picture are at the bottom of each triad.

Four types of triad were presented to children (see Figure 1):

(i) Food-color triads: triads with fruits or vegetables as target pictures and test picture 2 matched for color $(n=3$, see Figure 1a)

(ii) Food-shape triads: triads with fruits or vegetables as target pictures and test picture 2 matched for shape $(n=4$, see Figure $1 \mathrm{~b})$

(iii) Artifact-color triads: triads with artifacts as target pictures and test picture 2 matched for color $(n=3$, see Figure 1c)

(iv) Artifact-shape triads: triads with artifacts as target pictures and test picture 2 matched for shape $(n=4$, see Figure $1 \mathrm{~d})$.

For each triad presented successively, children were told a property of the target picture and then were asked to generalize this property to one of the two test pictures. To avoid any 
background knowledge effect that might vary from one child to another, unknown stimuli

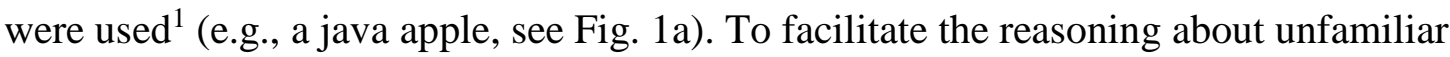
stimuli (Jaswal, Lima, \& Small, 2009; Macario, 1991), we used a puppet named 'T'choupi', which also avoided the risk of a transfer of children's food preferences or consumption habits to the task (T'choupi' is a well-known non-human cartoon character). For the food-color triads and food-shape triads children were told (translated from the French): "T'choupi will come to my place to have dinner, but I don't know what he eats. You have to help me find out. T'choupi eats this \{pointing to the target picture\}. What else does T'choupi eat, this or this \{pointing to the test pictures\}?"

For the artifact-color triads and artifact-shape triads, the instruction was as follows (translated from the French): “T'choupi will come to my place to play, but I don't know what he plays with. You have to help me find out. T'choupi plays with this \{pointing to the target picture\}. What else does T'choupi play with, this or this \{pointing to the test pictures\}?" Presentation order of the triads was randomized with the constraint that all the food-color triads and food-shape triads were grouped together as well as the artifact-color triads and artifact-shape triads. Within each triad the position of test picture 1 and test picture 2 were also randomized. Two additional triads composed of clothing and artifacts were presented beforehand as training for participants.

The experimenter recorded the children's responses to each of the 14 triads. A score of 1 was given when children chose test picture 1 (category-based response) and a score of 0 was given when the participant chose test picture 2 (perceptual-based response).

\footnotetext{
${ }^{1}$ We validated the unfamiliarity of the stimuli used in the study with an external adult sample $(n=20)$. Participants were asked whether they recognize each of the 42 pictures used in the stimuli set. Each participant recognized significantly less than $10 \%$ of the pictures (all $p \mathrm{~s}>0.065$ from binomial tests, with 42 trials and $\mathrm{p}=$ $0.1)$.
} 
Then for each type of triads, the scores were summed across all triads ( 3 or 4 ) and divided by the number of triads, to obtain four induction scores for each participant (ranging from 0 to 1).

\section{Discrimination Task}

Following the induction task, children participated in a discrimination task. This second task served to control for the children's capacity to discriminate between the unknown food items and the minerals used as color or shape matches in the food triads ${ }^{2}$. The stimuli were 14 pictures extracted from the food-color triads and food-shape triads ( 7 unknown fruits or vegetables and 7 minerals). Each item was printed on a separate laminated paper measuring $10.5 \times 14.8 \mathrm{~cm}$. The pictures were controlled for size, contrast, and luminosity.

Discrimination capacities were evaluated in a forced sorting task paradigm: children were asked to sort the items into two distinct boxes. All the items were arranged in front of the child with the following instructions (translated from the French): "Now I need you to sort the cards into two boxes. In this box, you put the things that can be eaten; in this box you put the other things (to avoid negative wording). I have to first lay all the cards on the table and then you can start."

The order in which the stimuli were arranged in front of the participant was randomized, as was the position of the boxes on the table.

The experimenter recorded for each child the number of hits, correct rejections, misses, and false alarms. Hit and correct rejection correspond to a correct categorization of the pictures (hit: an edible item placed in the 'things that can be eaten' box; correct rejection: an inedible item placed in the 'other things' box), whereas miss and false alarm correspond to a wrong

\footnotetext{
${ }^{2}$ Given the short attention spans of the 3- to 4-year-old children, we could not assess their capacity to discriminate between the unknown artifact stimuli and the minerals used as color or shape matches.
} 
categorization (miss: an edible item placed in the 'other things' box; false alarm: an inedible item placed in the 'things that can be eaten' box). Based on these scores we computed two psychophysical indices: the discriminability index A and the bias index B, derived from signal detection theory (Macmillan \& Creelman, 2004), but adapted to the needs of experiments with low numbers of stimuli (successfully used in Morin-Audebrand, Mojet, Chabanet, Issanchou, Møller, Köster, \& Sulmont-Rossé, 2012);

- $\quad$ Discriminability index $=\log \left[\left(\mathrm{N}_{\mathrm{H}}+0.5\right) /\left(\mathrm{N}_{\mathrm{MS}}+0.5\right)\right]-\log \left[\left(\mathrm{N}_{\mathrm{FA}}+0.5\right) /\left(\mathrm{N}_{\mathrm{CR}}+0.5\right)\right]$

- $\quad$ Bias index $=-\log \left[\left(\mathrm{N}_{\mathrm{H}}+\mathrm{N}_{\mathrm{FA}}+0.5\right) /\left(\mathrm{N}_{\mathrm{MS}}+\mathrm{N}_{\mathrm{CR}}+0.5\right)\right]$

Here $\mathrm{N}_{\mathrm{H}}, \mathrm{N}_{\mathrm{CR}}$, $\mathrm{N}_{\mathrm{MS}}$ and $\mathrm{N}_{\mathrm{FA}}$ represent the number of hits, correct rejections, misses and false alarms respectively. In our study, the discriminability index A could range from -2.35 to 2.35, and a score superior to 0 indicates that a participant provided more correct answers (hit and correct rejection) than wrong answers (miss and false alarm). The bias index B could range from - 1.46 to 1.46 . In this case, a score superior to 0 shows a response tendency toward inedibility (the participant tends to put more pictures in the 'other things' box) whereas a score inferior to 0 shows a response tendency toward edibility (the participant tends to put more pictures in the 'things that can be eaten' box).

\section{Statistical analyses}

To test our two hypotheses, we fit seven generalized linear models to the data with the induction scores as the outcome measure. The models were constructed by iteratively adding possible predictive variables to the null model (M0; containing just the intercept and any predictors). The possible predictive variables were the type of triad (food-color, food-shape, artifact-color, and artifact-shape), food neophobia scores, food pickiness scores, psychophysical indices A and B, and children's age. The interaction between the type of triad and food neophobia scores was also added to the models. The possible predictive variables 
were retained in the model if their addition led to a significant decrease of the AIC (Akaike Information Criterion, $\mathrm{Hu}, 2007)$, as attested by a chi-squared test. For instance, the predictive variable "type of triad" was selected because the model with this variable (model M1, see Table 2) better fit the data (i.e., has a significantly lower AIC) than the model without this variable (model M0, see Table 2). If there was no decrease of the AIC, the predictive variables were left out of the following iteration. For instance, the predictive variable "bias index B" was not selected because the model with this variable (model M6, see

Table 2) did not better fit the data than the model without this variable (model M5, see Table 2). All statistical analyses were conducted using R version 3.4.0 (R Core Team, 2017) running on RStudio version 1.0.143 (R Studio Team 2016) and the following four packages were used: lme4 (Bates, Maechler, Bolker, \& Walker, 2015), MuMIn (2016), lmerTest (Kuznetsova, Brockhoff, \& Christensen, 2016), and multcomp (Bretz, Hothorn, \& Westfall, 2016).

\section{Results}

Overall, children's induction scores were above chance level (as attested by a Wilcoxon test, $M=0.62, s d=0.17, V=4073.5, p<.0001$, see Table 1). Children tended to generalize the property of the target picture to the test picture belonging to the same taxonomic superordinate category. The same pattern was found for each type of triad (see Table 1). Additionally, children' discrimination index A was significantly above $0(M=0.21, s d=$ $0.52 ; V=2014, p<.01)$. They were able to sort edible items from non-edible items correctly. Children' bias index B was significantly below $0(M=-0.48, s d=0.68 ; V=516, p<.01)$. They had a rather liberal sorting tendency, considering most of the items edible. 
Table 1. Mean induction score and standard deviation for each triad type.

\begin{tabular}{lll}
\hline Triad & Mean & Standard Deviation \\
\hline food-color triad & $0.62 * * *$ & 0.29 \\
food-shape triad & $0.59 * *$ & 0.25 \\
artifact-color triad & $0.71 * * *$ & 0.28 \\
artifact-shape triad & $0.58 *$ & 0.27 \\
Overall & $0.62 * * *$ & 0.17 \\
\hline Note. $*$ for $p<.01 ; * *$ for $p<.001 ; * * *$ for $p<.0001$. Test reported in the Table are
\end{tabular}

Wilcoxon tests to examine whether children's induction scores were above chance level. $P$ values are corrected for multiple tests (significance level is at 0.01).

The seven generalized linear models are displayed in Table 2, which shows that M5 was the best model in view of the data, because it had the lower AIC. The predictive variables we retained in this model were the type of triad (food-color, food-shape, artifact-color, and artifact-shape), food neophobia score, and discriminability index A. 
Table 2. Goodness of fit of the Generalized Linear Models.

\begin{tabular}{|c|c|c|c|c|c|c|}
\hline \multicolumn{2}{|c|}{ Model } & \multirow{2}{*}{$\begin{array}{l}D f \\
1\end{array}$} & \multirow{2}{*}{$\begin{array}{l}\text { AIC } \\
113.18\end{array}$} & \multirow[t]{2}{*}{ Deviance } & \multicolumn{2}{|c|}{ Pseudo $R^{2} p$ value } \\
\hline M0 & 1 & & & & 0.171614 & \\
\hline M1 & $\ldots+$ type of triad & 3 & 109.45 & 9.74 & 0.1782584 & $.02096^{*}$ \\
\hline M2 & $\ldots+$ type of triad + neophobia & 4 & 107.91 & 3.54 & 0.1782794 & $.06006^{\bullet}$ \\
\hline M3 & $\ldots+$ type of triad + neophobia + pickiness & 5 & 108.81 & 1.10 & 0.1782862 & 0.2943 \\
\hline M4 & $\ldots+$ type of triad $*$ neophobia & 4 & 113.78 & 0.13 & 0.1786078 & 0.9879 \\
\hline M5 & $\ldots+$ type of triad + neophobia $+\mathrm{A}$ & 5 & 106.30 & 3.61 & 0.1783002 & .05748 \\
\hline M6 & $\ldots+$ type of triad + neophobia $+\mathrm{A}+\mathrm{B}$ & 6 & 108.24 & 0.068 & 0.1783006 & .7943 \\
\hline M7 & $\ldots+$ type of triad + neophobia $+\mathrm{A}+$ age & 6 & 108.18 & 0.12 & 0.1783009 & 0.7253 \\
\hline
\end{tabular}

Note. The Deviance column indicates the increase in explained variability. For example, the residual deviance for $M 1$ was 95.449, while the residual deviance for M2 was 91.913, a difference of 3.54. * for $p<.05 ; \cdot$ for $p<.1$.

First, we observed a significant effect of the type of triad on the induction score, $F(3,109)=$ $6.19, p=0.00041$. Post-hoc Tukey comparisons ( $\mathrm{p}$-value corrected for multiple comparisons) revealed that children had a significantly higher score for the artifact-color triads $(M=0.71$, $s d=0.28, p=0.037)$ than for the food-color triads $(M=0.62, s d=0.29$; see Fig. 2$)$. Additionally, children scored significantly higher for the artifact-color triads $(M=0.62, s d=$ $0.29)$ than the artifact-shape triads $(M=0.58, s d=0.27, p<.0001$; see Fig. 2). Finally, children scored significantly higher for the artifact-color triads $(M=0.71, s d=0.28)$ than for the food-shape triads $(M=0.059, s d=0.25, p=0.0022$, see Fig. 2$)$. 
Figure 2. Mean induction scores for the triads in each condition of the induction task.

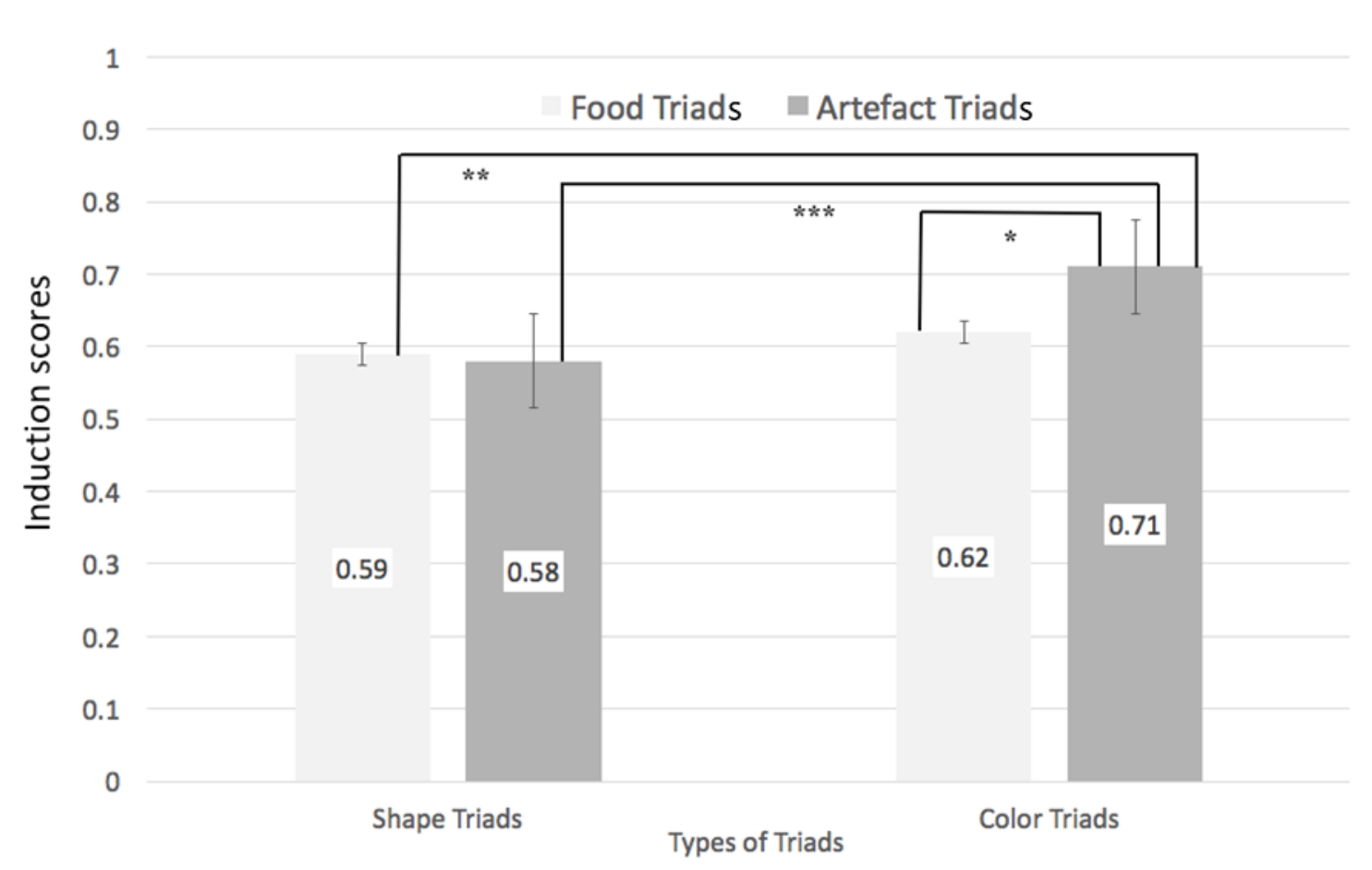

Note. Significant differences between the types of triads are marked: * for $p<.05 ; * *$ for $p$ $<.01 ; * * *$ for $p<.001$.

Second, a significant effect of neophobia was found, $\mathrm{F}(1,109)=3.93, p=0.049$. Food neophobia scores and induction scores were significantly negatively correlated (as attested by Kendall's tau $=-0.18, p=.011)$. Children scoring highly on the food neophobia subscale performed more poorly on the induction task than the other children.

Finally, the model M5 revealed an near significant effect of the discriminability index A $(\mathrm{F}(1,109)=3.9366, p=.058)$. Discriminability indices A and induction scores were significantly positively correlated (as attested by Kendall's tau $=0.15, p=0.034$ ). Children having a high discriminability index A tended to score higher in the induction task. 


\section{Discussion}

\section{The contributions of shape and color to both food and artifact categorization and}

\section{induction}

The results partly confirmed Hypothesis 1 . Indeed, the mean score on the triads in which the taxonomic category membership was pitted against color-similarity was significantly lower when the target image was a food than when it was an artifact (see Fig. 2). This result is in line with the claim that food inductive reasoning is domain specific in the sense that the range of inputs to which food inductive reasoning is sensitive differs from the range of inputs to which other types of inductive reasoning are sensitive. However, this interpretation should be taken cum grano salis. No significant difference was obtained between the mean score on the food-shape triads and the mean score on the food-color triads. In contrast a significant difference in the mean scores for the artifact-shape and artifact-color triads was revealed: children's performance was significantly better on artifact-color triads than artifact-shape triads.

Nevertheless, our results invite us to reconsider Macario's claim (1991) that children tend to use color rather than shape to generalize the edibility property (i.e. being food for the puppet) to novel food items. One possibility is that this discrepancy, between Macario's conclusion and the results of the present experiment, is due to a difference in the food stimuli used. Indeed, Macario used unidentifiable abstract objects made from paraffin wax, presented as food by the experimenter, whereas we used pictures of existing but unknown foods. Moreover, we presented the unknown foods in their entirety. The children may thus have tried to relate the unknown food stimuli to familiar food prototypes because of overall shape similarities (for instance the shape of the java apple was close to the prototypical shape of pears). Unsurprisingly, this type of similarity cannot serve as a reliable guide for inductive reasoning when the food items are cut or transformed (except for canonically shaped 
processed food such as burgers, ice-cream, etc.), or a fortiori when the food stimuli are abstract objects presented as puppet food as in Macario's experiment. A promising approach would thus be to vary the degree of transformation of different food stimuli to determine to what extent it impacts the contribution of color and shape cues to food categorization, induction and acceptance.

\section{The relationship between food rejection and inductive reasoning}

The present experiment partly confirmed the previously reported negative relationship between food rejection and inductive reasoning performance. However, only the second dimension of the CFRS scale, which captures food neophobia, significantly correlated with children's induction performance and has been retained in the model M5. No significant effect was obtained regarding food pickiness (contrary to Rioux, et al., 2017b). A likely reason why only food neophobia influenced induction performance dwells in the nature of the food stimuli. Contrary to the study conducted by Rioux and colleagues (Ibidem), the food stimuli in the food triads were all unfamiliar and presumably unknown to the children. It is thus reasonable to hypothesize that these stimuli more easily triggered the food neophobia disposition which is generally defined as the fear of novel food. On the contrary, since food pickiness is not straightforwardly related to novelty, it is not surprising that the unknown food stimuli triggered this disposition only to a lesser extent.

\section{Food neophobia negatively impacted inductive reasoning performance in both the food and artifact domains}

The results of the present study failed to confirm Hypothesis 2. Children scoring highly on the food neophobia subscale performed poorly on the induction task for all triads, compared to their more neophilic counterparts (no interaction between neophobia and triad type has 
been retained in the linear model M5). Interestingly, the idea that the study failed to confirm Hypothesis 2 simply because the children could not recognize the food items as such is unlikely, given the results obtained in the discrimination task. Indeed, the results of the discrimination task confirmed that the children could discriminate the food stimuli from the minerals that we used as color or shape matches.

From a theoretical point of view, the fact that Hypothesis 2 was not confirmed and that the results go rather in the opposite direction is important because it suggests that food neophobia could be a particular instance of a more domain-general disposition to fear novelty. This interpretation is in line with a recent study that showed that (4.5-year-old) children's negative affect, such as fear, toward novel artifacts featured among the predictors of both dispositional and behavioral food neophobia (Moding \& Stifter, 2016).

\section{Limits and perspectives}

To summarize, our results provided the first evidence that food neophobia influences inductive reasoning performance in both the food and artifact domains. The results also reinforced previous findings by showing that children were more likely to use shape than color to generalize a functional property (i.e. being a toy for the puppet) to novel artifacts. However, they also call into question the assumption that in the food domain, color would have better predictive validity than shape.

We acknowledge that the present study has some limitations. Primarily we only conducted the discrimination task on the unknown food stimuli and the minerals we used as distractors. We could not conduct a second discrimination task for artifacts versus minerals because it would have been too demanding for our participants after the inductive reasoning task. The consequence of course is that we cannot be certain that the children were perfectly able to 
discriminate between the artifacts and minerals that we used as either color or shape associates. Secondly, we did not assess children's temperament, to avoid caregivers drop off of the study because of too long questionnaires. Nevertheless recent studies have revealed that children's negative affect during novel artifact exploration has a predictive effect on their neophobia (Moding \& Stifter, 2016) and that children with high tactile defensiveness were likely to be more picky (Nederkoorn, Jansen, \& Havermans, 2015). An important next step will be then to assess children's temperament, with for instance the short version of the Children Behavior Questionnaire (short CBQ, Putnam \& Rothbart, 2006), to investigate whether children's temperament influence the relationship between food rejection and induction strategies.

From a more applied perspective, such an exploratory study on the potential domain specificities of the food learning mechanisms and their sensitivity to individual factor such as food neophobia represents a necessary preliminary step toward a new type of more efficient food education programs. Indeed, the promotion of dietary diversity from early age has been tackled through the use of a variety of interventions generally aiming at promoting fruit and vegetable acceptance in children and decreasing food rejection (i.e. food neophobia and pickiness) (Appleton, et al., 2016; Evans, Christian, Cleghorn, Greenwood, \& Cade, 2012; Keller, 2014; Knai, Pomerleau, Lock \& McKee, 2006; Perez-Rodrigo \& Aranceta, 2001). A vast majority of the existing interventions capitalize on one mechanism: the mere exposure effect. This mechanism has been generally described as follow: the exposition to one instance of a given food stimulus is sufficient to trigger a more positive attitude toward a subsequent instance of that particular stimulus, because it increases the familiarity with it (Zajonc, 1968). However, this type of approach is disconnected from children's actual food learning mechanisms and incipient knowledge about food. Perhaps, this is the reason why the majority 
of these studies have reported only a relative success and generally failed to demonstrate that sustainable behavioral changes have been induced in the targeted population (Appleton et al., 2016).

Recently, an alternative way of designing interventions aiming at fostering dietary variety has led to very encouraging results. These new interventions are characterized by the fact that they did not seek behavioral change per se but rather aim first at improving the conceptual apparatus of young children. Indeed, such interventions capitalized on children's early food knowledge which, once properly enriched, bootstrapped (Carey, 2009) or boosted (GrüneYanoff \& Hertwig, 2015), impacts positively and more sustainably children food behavior and preferences (Grisphover \& Markman, 2013; Nguyen, McCullough, \& Noble, 2011; Thibaut, Nguyen, Murphy, 2016). For instance, Grisphover \& Markman (2013) compared a usual educational program about nutrition with a program that adopted a knowledge-based approach, giving children a rich conceptual framework about food. These authors found that children who attended the latter program ate more vegetables at snack time. Similar findings were found by Nguyen and colleagues (2011), using a comparable theory-based education program (see also Dejesus, Shutts \& Kinzler, 2017, on the influence of the knowledge of others's food preferences on children food behaviors). However, to boost children's incipient knowledge about food and the related capacities such as categorization and induction, it is of crucial importance to take into account the individual factors that may modulate children's conceptual abilities. Indeed, on the basis of the present results we may seriously question the relevance of an intervention deployed equally on all the children of a same age group since the maturity of their conceptual system about food depends also upon the intensity of their individual neophobic tendencies. Furthermore, to enrich children's conceptual knowledge about food it is crucial to select the relevant inputs. Such a proper selection of the range of inputs relevant for the enrichment of the early food concepts cannot be achieved without a 
thorough investigation of potential domain-specific constraints on conceptual abilities about food. For instance, Rioux and colleagues (2017b) obtained results suggesting that mature inductive reasoning strategies in the food domain rely rather on category membership whereas incipient inductive strategies (observable in young and/or neophobic children) are more driven by color-similarity. They interpreted these results as suggesting that as inductive reasoning system improves, the color cues tend to lose their predictive validity in favor of more conceptual pieces of information. In another experiment (Rioux, Lafraire, Picard, 2018), they exploited these results to design an intervention targeting preschoolers. They thus exposed children to many exemplars of the same basic level categories (zucchini, tomatoes, apple, etc.) varying in color. The hypothesis behind that intervention in which they varied systematically the color within each basic level food categories was to decrease the predictive validity of the color cues in the food domain to force the transition to more developed inductive reasoning strategies relying on category membership. The results they obtained were mitigated. However, the results of the present experiment by revealing an unsuspected contribution of the shape cues to inductive reasoning in the food domain may feed a new attempt to boost the food categories system. Indeed, a promising perspective would be to vary both the color and shape of the exemplars of different basic level food categories and to compensate the increased state of uncertainty by providing extra pieces of conceptual information whose efficiency has been recently demonstrated by Gripshover \& Markman (2013) and Nguyen and colleagues (2011). Such an approach will be perfectly in line with a promising trend for designing educational policies according to which such policies should be anchored in evidence about the developmental and the domain specificities of the learning mechanisms and the knowledge structures at hand (Albus et al., 2007; Bruer, 1993; Meltzoff et al., 2009). 


\section{Acknowledgments}

The authors would like to acknowledge the financial support they received from the Research Center of the Institut Paul Bocuse. We also would like to thank Gratianne Dumas and Lyon municipality who helped us recruit participants, Camille Lakhlifi who contributed to the fieldwork, the schools of Ecully, Lyon, and Paris for their warm welcome, parents for their trust, and children who spared no efforts and did their best.

\section{References}

Albus, J. S., Bekeley, G. A., Holland, J. H., Kanwisher, N. G., Krichmar, J. L., Mishkin, M., et al. (2007). A proposal for a Decade of the Mind Initiative. Science, 317, 1321.

Appleton, K. M., Hemingway, A., Saulais, L., Dinnella, C., Monteleone, E., Depezay, L., ... \& Hartwell, H. (2016). Increasing vegetable intakes: rationale and systematic review of published interventions. European journal of nutrition, 55(3), 869-896.

Bates, D. Maechler, M., Bolker, B. \& Walker, S. (2015). Fitting Linear Mixed-Effects Models Using lme4. Journal of Statistical Software, 67(1), 1-48.

Birch, L. L., \& Fisher, J. O. (1998). Development of eating behaviors among children and adolescents. Pediatrics, 101(Supplement 2), 539-549.

Bretz, F., Hothorn, T., \& Westfall, P. (2016). Multiple comparisons using R. CRC Press.

Bruer, J. (1993). Schools for thought: A science of learning in the classroom. Cambridge, MA: MIT Press.

Carey, S. (2009). The origin of concepts. Oxford University Press.

Chomsky, N. (1980). Rules and representations. New York: Columbia University Press. 
Dawkins, R. (1976). The selfish gene. New York: Oxford University Press.

DeJesus, J. M., Shutts, K., \& Kinzler, K. D. (2017). Mere social knowledge impacts children's consumption and categorization of foods. Developmental Science, e12627.

Dovey, T. M., Staples, P. A., Gibson, E. L., \& Halford, J. C. (2008). Food neophobia and 'picky/fussy’ eating in children: a review. Appetite, 50(2), 181-193.

Evans, C. E., Christian, M. S., Cleghorn, C. L., Greenwood, D. C., \& Cade, J. E. (2012). Systematic review and meta-analysis of school-based interventions to improve daily fruit and vegetable intake in children aged 5 to $12 \mathrm{y}-$. The American journal of clinical nutrition, 96(4), 889-901.

Falciglia, G. A., Couch, S. C., Gribble, L. S., Pabst, S. M., \& Frank, R. (2000). Food neophobia in childhood affects dietary variety. Journal of the American Dietetic Association, 100(12), 1474-1481.

Fodor, J. A. (1983). The modularity of mind: An essay on faculty psychology. MIT press.

Foroni, F., Pergola, G., \& Rumiati, R. I. (2016). Food color is in the eye of the beholder: the role of human trichromatic vision in food evaluation. Scientific reports, 6, 37034.

Foroni, F., Rumiati, R. (2017) Food Perception and Categorization: From Food/No-Food to Different Types of Food. In Cohen, H., \& Lefebvre, C. (Eds.). (2005). Handbook of categorization in cognitive science. Elsevier.

Gelman, R. (1990). Structural constraints on cognitive development: Introduction to a special issue of Cognitive Science. Cognitive Science, 14(1), 3-9.

Gelman, S. A., \& Markman, E. M. (1986). Categories and induction in young children. Cognition, 23(3), 183-209. 
Gelman, S. A. (1988). The development of induction within natural kind and artifact categories. Cognitive psychology, 20(1), 65-95.

Gelman, S. A. (2004). Psychological essentialism in children. Trends in cognitive sciences, 8(9), 404-409.

Gigerenzer, G., \& Goldstein, D. G. (2011). The recognition heuristic: A decade of research. Judgment and Decision Making, 6(1), 100.

Gripshover, S. J., \& Markman, E. M. (2013). Teaching young children a theory of nutrition: Conceptual change and the potential for increased vegetable consumption. Psychological Science, 24(8), 1541-1553.

Grüne-Yanoff, T., \& Hertwig, R. (2016). Nudge versus boost: How coherent are policy and theory?. Minds and Machines, 26(1-2), 149-183.

Guinard, J.-X. (2000). Sensory and consumer testing with children. Trends in Food Science and Technology, 11(8), 273-283.

Hirschfield, L. A., \& Gelman, S. A. (1994). Mapping the mind. Cambridge: MA, Cambridge.

Hu, S. (2007). Akaike information criterion. Center for Research in Scientific Computation, 93.

Jaswal, V. K., Lima, O. K., \& Small, J. E. (2009). Compliance, conversion, and category induction. Journal of experimental child psychology, 102(2), 182-195.

Keil, F. (1981). Constraints on knowledge and cognitive development. Psychological Review, 88(3), 197-227.

Keller, K. L. (2014). The use of repeated exposure and associative conditioning to increase vegetable acceptance in children: explaining the variability across studies. Journal of the Academy of Nutrition and Dietetics, 114(8), 1169-1173. 
Knai, C., Pomerleau, J., Lock, K., \& McKee, M. (2006). Getting children to eat more fruit and vegetables: a systematic review. Preventive medicine, 42(2), 85-95.

Kuznetsova, A., Brockhoff, P. B., \& Christensen, R. H. (2016). B (2016). lmerTest: Tests in linear mixed effects models. R package version 2.0-32.

Lafraire, J., Rioux, C., Giboreau, A., \& Picard, D. (2016). Food rejections in children: Cognitive and social/environmental factors involved in food neophobia and picky/fussy eating behavior. Appetite, 96, 347-357.

Laureati, M., Bergamaschi, V., \& Pagliarini, E. (2015). Assessing childhood food neophobia: validation of a scale in Italian primary school children. Food Quality and Preference, 40(A), 8-15.

Lavin, T. A., \& Hall, D. G. (2001). Domain effects in lexical development: Learning words for foods and toys. Cognitive Development, 16(4), 929-950.

Macario, J. F. (1991). Young children's use of color in classification: Foods and canonically colored objects. Cognitive Development, 6(1), 17-46.

Macmillan, N. A., \& Creelman, C. D. (2004). Detection theory: A user's guide. Psychology press.

Mahon, B. Z., \& Caramazza, A. (2011). What drives the organization of object knowledge in the brain?. Trends in cognitive sciences, 15(3), 97-103.

Marr, D. (1982). Vision. San Francisco, CA: Freeman.

Meltzoff, A. N., Kuhl, P. K., Movellan, J., \& Sejnowski, T. J. (2009). Foundations for a new science of learning. Science, 19, 284-288.

Moding, K. J., \& Stifter, C. A. (2016). Stability of food neophobia from infancy through early childhood. Appetite, 97, 72-78. 
Morin-Audebrand, L., Mojet, J., Chabanet, C., Issanchou, S., Møller, P., Köster, E. P., \& Sulmont-Rossé, C. (2012). The role of novelty detection in food memory. Acta psychologica, 139(1), 233-23

MuMIn, B. K. (2016). Multi-model inference. R package version 1.15. 6. 2016.

Murphy, G. (2002). The big book of concepts. MIT press.

Nederkoorn, C., Jansen, A., \& Havermans, R. C. (2015). Feel your food. The influence of tactile sensitivity on picky eating in children. Appetite, 84, 7-10.

Nguyen, S. P., McCullough, M. B., \& Noble, A. (2011). A theory-based approach to teaching young children about health: A recipe for understanding. Journal of Educational Psychology, 103(3), 594-606.

Pérez-Rodrigo, C., \& Aranceta, J. (2001). School-based nutrition education: lessons learned and new perspectives. Public Health Nutrition, 4(1a), 131-139.

Pliner, P. (1994). Development of measures of food neophobia in children. Appetite, 23(2), $147-163$.

Pliner, P., \& Hobden, K. (1992). Development of a scale to measure the trait of food neophobia in humans. Appetite, 19(2), 105-120.

Putnam, S. P., \& Rothbart, M. K. (2006). Development of short and very short forms of the Children's Behavior Questionnaire. Journal of personality assessment, 87(1), 102112.

R Core Team (2017). R: A language and environment for statistical computing. R Foundation for Statistical Computing, Vienna, Austria. URL https://www.R-project.org/. 
Rioux, C., Picard, D., \& Lafraire, J. (2016). Food rejection and the development of food categorization in young children. Cognitive Development, 40, 163-177.

Rioux, C., Lafraire, L., \& Picard, D. (2017a). The Children Food Rejection Scale: Development and validation of a new scale to assess food neophobia and pickiness among 2- to 7-years old French children. European Review of Applied Psychology, $67,67-77$.

Rioux, C., Lafraire, J., \& Picard, D. (2017b). Food rejection and the development of food category-based induction in 2-6 years old children. Journal of Cognitive Psychology, $1-13$

Rioux, C., Lafraire, J., \& Picard, D. (2018). Visual exposure and categorization performance positively influence 3-to 6-year-old children's willingness to taste unfamiliar vegetables. Appetite, 120, 32-42.

Rozin, P. (1976). The selection of foods by rats, humans, and other animals. In Advances in the Study of Behavior (Vol. 6, pp. 21-76). Academic Press.

Rozin, P. (1979). Preference and affect in food selection. Preference behavior and chemoreception, 289-302.

Rumiati, R. I., \& Foroni, F. (2016). We are what we eat: How food is represented in our mind/brain. Psychonomic bulletin \& review, 23(4), 1043-1054.

R Studio Team (2016). RStudio: Integrated Development for R. RStudio, Inc., Boston, MA URL http://www.rstudio.com/.

Spelke, E. S., \& Kinzler, K. D. (2007). Core knowledge. Developmental science, 10(1), 8996. 
Sperber, D., \& Hirschfeld, L. A. (2004). The cognitive foundations of cultural stability and diversity. Trends in cognitive sciences, 8(1), 40-46.

Taylor, C. M., Wernimont, S. M., Northstone, K., \& Emmet, P. M. (2015) Picky/fussy eating in children: Review of definitions, assessment, prevalence and dietary intakes. Appetite, 95, 349-359.

Thibaut, J.-P., Nguyen, S. P., \& Murphy, G. L. (2016). Body and soul: Do children distinguish between foods when generalizing biological and psychological properties? Early Education and Development, 27(8), 1-13.

Wertz, A. E., \& Wynn, K. (2014a). Selective social learning of plant edibility in 6-and 18month-old infants. Psychological science, 25(4), 874-882.

Wertz, A. E., \& Wynn, K. (2014b). Thyme to touch: Infants possess strategies that protect them from dangers posed by plants. Cognition, 130(1), 44-49.

Zajonc, R. B. (1968). Attitudinal effects of mere exposure. Journal of personality and social psychology, 9(2p2), 1. 